\title{
Abstract: Patches in Magnetic Particle Imaging
}

Mandy Ahlborg ${ }^{1}$, Christian Kaethner ${ }^{1}$, Patryk Szwargulski ${ }^{2,3}$, Tobias Knopp ${ }^{2,3}$, Thorsten M. Buzug ${ }^{1}$

\author{
${ }^{1}$ Institute of Medical Engineering, Universität zu Lübeck \\ ${ }^{2}$ Section for Biomedical Imaging, University Medical Center Hamburg-Eppendorf \\ ${ }^{3}$ Institute for Biomedical Imaging, Hamburg University of Technology \\ ahlborg@imt. uni-luebeck.de
}

Magnetic Particle Imaging (MPI) is a tracer-based imaging technology [1] with which superparamagnetic nanoparticles can be detected and located using specific magnetic fields. The selection field, a gradient field in form of a field free point (FFP), restricts the area in which particles can be remagnetized. The drive field, a homogeneous and time-varying magnetic field, remagnetizes the particles and moves the FFP. As a result a time dependent signal can be measured and then reconstructed to the actual spatial distribution of the tracer.

The movement of the FFP is specified by the frequency and the amplitude of the drive field and is referred to as trajectory. The ratio of amplitude and gradient particularly defines the size of the field of view (FOV) of a trajectory. In theory, the trajectory size can be chosen arbitrarily to meet the requirements of the (medical) imaging application. However, technical as well as physiological aspects limit the actual amplitude of the drive field and thus the FOV size. A possible solution to this constraint are imaging patches. Patches are multiple FOVs at different positions that need to be combined to obtain one large image.

In [2], the influence of overlaps between patches and different reconstruction techniques were investigated. The main focus was the reduction of truncation artifacts that occur on the edges of individual patches. It was shown that it is necessary to choose the MPI system matrix larger than the applied trajectory resulting in an inherent system matrix overlap. Further, a small trajectory overlap between patches significantly reduces artifacts. The data redundancy resulting from the overlapping areas has to be considered in the image reconstruction step. The two superior methods identified in [2] are (i) reconstruction of the individual patches and consecutive combination of the images by cutting off the patch edges and (ii) combination of the patch data in one system of equations and joint reconstruction.

In this contribution, we will validate the results of [2] with different experimental settings to demonstrate the robustness of the proposed methods.

\section{References}

1. Gleich B, Weizenecker J. Tomographic imaging using the nonlinear response of magnetic particles. Nature. 2005;435(7046):1214-1217.

2. Ahlborg M, Kaethner C, Knopp T, et al. Using data redundancy gained by patch overlaps to reduce truncation artifacts in magnetic particle imaging. Phys Med Biol. 2016;61(12):4583. 